\title{
Inspection of the Scene at the Initial Stage of the Investigation of Criminal Violations of Safety Rules during Mining
}

\author{
Oleg Sergeev ${ }^{1, *}$, Olga Safargalieva $^{1}$, and Yuriy Volgin ${ }^{1}$ \\ ${ }^{1}$ Kemerovo State University, 650000, Krasnaya St. 6, Russia
}

\begin{abstract}
The article examines the features of tactics for inspecting the scene of the incident when investigating criminal violations of safety rules during mining. When observing the preparatory stage, special attention in the inspection of the scene is paid to the membership. In particular, we believe that when investigating criminal violations of safety rules in the conduct of mining operations, it is inappropriate to involve attesting witnesses in the inspection. At the same time, it is advisable to expand the circle of specialists involved in the inspection of the accident site (electricians, mine surveyors, etc.). A feature of the tactics of the working stage, in our opinion, is the use of an eccentric research method, regardless of the type of accident. At the same time, when directly searching for traces, carriers of information, moving from the center to the periphery, one should be guided, first of all, by the type of man-triggered incident. The final stage consists of the following: a) finalization of the results of the inspection of the scene of the accident (we recommend that forensic experts be involved in this work - if there are corpses, and specialists of technical profiles corresponding to the type of accident; $b$ ) putting forward versions about the essence of what happened, its reasons, the perpetrators and determining the direction of the investigation.
\end{abstract}

\section{Introduction}

Ensuring the safety of mining operations is a prerequisite for the sustainable development of the region. Therefore, timely and high-quality investigation of criminal violations of safety rules is important. Inspection of the scene as an urgent investigative action is aimed at establishing, researching and describing the situation at the scene of the incident, traces of the crime and other factual data, which, together with other evidence, make it possible to draw a conclusion about the event [1] and the mechanism of the crime. The urgency of its implementation is due to the following: the danger of irreversible changes in the initial situation and objects undergoing inspection, as a result of eliminating the consequences of the accident; the adoption by interested parties of measures aimed at the destruction or concealment of the traces of the crime; information richness of the scene and the available opportunity to obtain in the process of its implementation such information that cannot be obtained during other investigative actions [2].

*Corresponding author: Oleg.s-v@mail.ru 
The noted circumstances necessitate research and, respectively, the development of organizational and tactical recommendations that increase the efficiency of site inspections in the investigation of criminal violations of safety rules during mining operations. A feature of criminal violations of safety rules during mining operations is, as a rule, the presence of victims - the consequences of criminal activity [3]. Inspection and examination of the corpse (corpses), the traumas present on them, which make it possible to judge the mechanism of their causing and the causes of death, are carried out on the surface, which does not exclude the possibility of errors and makes it necessary to increase the reliability of the forensic scientist's judgment, develop recommendations for an organizational plan aimed at minimizing the negative consequences of possible errors.

\section{Materials and Methods}

The basis for the study of the features of the inspection of the scene during the investigation of criminal violations of safety rules in the conduct of mining operations were the legal sources: the Constitution of the Russian Federation [4], the Criminal Code of the Russian Federation [3], the Criminal Procedure Code of the Russian Federation [5], as well as departmental normative legal acts regulating the activities of enterprises carrying out mining operations. The empirical basis of the conclusions formulated in the article consists of materials of 102 criminal cases, 71 decisions on refusal to initiate a criminal case, the results of a questionnaire survey of employees of investigative departments and workers of the coal industry.

Research methods include the following: a) general research methods, such as formallogical; systemic structural analysis; b) specific research methods, such as comparative legal; modeling and statistical methods; questioning.

\section{Results and Discussion}

Inspection of the scene of the incident is the initial investigative action carried out "... in order to detect traces of a crime, to clarify other circumstances relevant to the case" (Article 176 of the Criminal Procedure Code of the Russian Federation). The significance of this investigative action is determined by the fact that at the scene, as a rule, there are numerous and varied material consequences of the crime in the form of various traces and other material evidences necessary for solving the crime. Therefore, the primary tasks of inspecting the scene of the incident in the investigated category of criminal cases should include:

1) Establishing and studying the very fact of a criminal violation of safety rules, and, in case of a positive result, deciding whether there has been a violation of safety rules.

2) Establishing the nature of specific violations of safety rules.

3) The establishment of the fire source.

All of the above tasks of inspecting the scene of the incident allow outlining the ways of disclosing a criminal violation of safety rules during mining and contribute to the conduct of preventive work.

Analysis of criminal cases initiated on the facts of criminal violations of safety rules during mining, and a questionnaire survey of law enforcement officials, led to the conclusion that the inspection of the scene was not carried out in all criminal cases. The inspection of the scene by the investigator was usually not carried out on the following grounds: a) the message came from the specialized inspectorates (the Federal Service for Environmental, Technological and Nuclear Supervision) and the investigator has the materials of the investigation of a special departmental commission. The materials received allow the investigator to understand the situation and nature of the incident, and decide on the initiation 
or refusal to initiate a criminal case. In addition, often, after the elimination of the accident and the completion of the commission's work, the situation at the scene of the accident, in whole or in part, due to objective reasons, changes; its original appearance is not preserved, which makes the forensic investigation of the scene of the accident difficult [2].

We have already considered ways of minimizing negative consequences in this situation, in particular, it is recommended that an investigator inspects the scene of the incident together with a departmental commission [2], especially if there is a real danger to the life and health of the members of the investigative-and-operational group. At the same time, the results of some studies indicate that investigators refuse to produce it even in the absence of objective reasons, underestimating the forensic value of the results of this investigative action and relying on the conclusions of specialized inspections, or conduct it superficially due to the lack of experience and knowledge of the features of the inspection of the scene of the incident on this categories of criminal cases.

A quality of inspection of the scene during the investigation of safety violations during mining depends on the clear organization of the preparatory measures. The leading role here is assigned to the investigator. He (she) organizes a trip to the scene, determines the composition of its participants, directs their actions at the scene, performs a set of actions to detect traces of the crime and describe the situation at the scene [6].

Preparatory measures should be carried out by the investigator even before entering the scene. Traditionally, the preparatory work to inspect the scene of the incident is divided into three stages: 1) before entering the scene; 2) on the surface of the mine; 3 ) before the start of the inspection, directly at the scene.

One of the main actions of the investigator at the first stage of the preparatory work is to determine the circle of participants in the inspection of the scene. The Criminal Procedure Law distinguishes between obligatory and optional participants in the examination. The obligatory ones include an investigator, attesting witnesses (except for the cases provided for in part 3 of article 170 of the Code of Criminal Procedure of the Russian Federation) and a forensic expert. Specialists and other participants in the inspection are optional.

If the situation at the scene allows witnesses to be involved in the investigative action, then the investigator, in our opinion, should invite the persons who understand the objects that are subject to inspection, but taking into account their interest in the results of the investigative action, i.e. persons, possibly working at this enterprise, but not associated with the emergency site. At the same time, we believe that literal adherence to the prescription of the law on the participation of attesting witnesses in the inspection of the scene (except for the cases provided for in part 3 of article 170 of the Criminal Procedure Code of the Russian Federation) in the considered category of criminal cases is undesirable for a number of reasons. These reasons are: the duration of the investigative action, an unfavorable emotional background, the presence of one's own attitudes and ideas about the essence of what happened and its causes can negatively affect both the objectivity of perception and the maintenance of the required level of attention. The absence of attesting witnesses can be successfully compensated by the use of technical means of recording, by expanding the circle of specialists - participants in the inspection of the scene of the incident.

The specifics of the mining industry, the unfamiliar and very specific for the investigator the situation in the coal mine, make it mandatory to invite as participants in the examination of various specialists, in addition to the traditional forensic expert. As such, specialists in various fields of mining can be invited (electrical, mining transport and ventilation specialists, mine surveyors, etc.). In addition, in our opinion, it is advisable for the investigator to involve the state labor inspector and (or) the State Mining Control (Gosgortechnadzor) inspector as specialists. These persons have special knowledge and can provide significant assistance to the investigator. However, it should be considered that these officials, if they supervise the object being inspected, may be interested in the outcome of the 
investigative action, and, therefore, in this case, their involvement as specialists is inappropriate.

In accordance with part 1 of article 178 of the Criminal Procedure Code of the Russian Federation, in all cases of examination of a corpse at the scene of an incident, the investigator must involve a forensic medical expert (or a doctor). The participation of a forensic expert in the inspection of the scene of the incident allows to establish not only the fact of the death of the victim, but also to establish individual circumstances of the incident, which are essential for determining the causes of the violation and the circumstances of the incident. However, practice shows that the ability of forensic doctors, even when examining the scene of the incident and the corpse at the place of its discovery, to explain the circumstances that are significant for the case are not in demand, since the examination of the scene by the investigator is practically not carried out due to various objective circumstances. Specialized departmental commissions, the materials of which are often used by the investigator to resolve the issue of initiating a criminal case, put forward versions and determine the direction of the investigation, also do not involve forensic doctors in the examination of the dead, but are limited only to sending the corpses for forensic medical research.

Along with specialists, the investigator, in some cases, can involve a representative of management of the enterprise where the accident occurred in the inspection of the scene of the accident, however, without involving persons interested in the outcome of the case.

At the stage of preparation for the inspection of the scene before entering it, in addition to determining the composition of the participants, the investigator must: 1) resolve the issue of providing the investigative and operational group with the technical means necessary to inspect the scene of the incident in the mines. This concerns the problems of lighting the object of inspection, the use of fire technical suitcases, gas analyzers and methods of recording the place of inspection on various media (photograph and video recording); 2) take measures to keep the scene of the incident unchanged. In order to implement this recommendation, he (she) instructs the management of the enterprise to take measures to protect the scene of the incident and prevent unauthorized persons there. However, given that in some cases the management may be interested in changing the situation at the scene, the investigator should simultaneously send police officers to guard the scene; 3 ) establish contact and interact with the departmental commission conducting the official investigation in a situation where the investigator begins work at the scene immediately after the incident [7].

At the second stage of preparation for the inspection of the scene of the incident - on the surface of the mine - the investigator must check the fulfillment of his instructions given before entering the scene, and concerning the provision of medical assistance to the victims and the protection of the scene of the incident. In case of non-fulfillment or improper fulfillment of orders, organize their fulfillment. Given the complexity of inspecting the accident site in a coal mine, the investigator must, before descending to the scene of the incident, familiarize himself with the working documentation for the emergency area and, if necessary, seizure it. For a good orientation at the scene of the incident, it is necessary to invite a surveyor to draw up a plot of the scene of the incident and clarify the route of movement to the place of the investigative action. Explain to all the participants in the inspection of their rights and obligations, distribute responsibilities between them and conduct appropriate safety briefing, for which to invite an employee of the enterprise who knows the emergency site, its specifics and is able to conduct this briefing sufficiently fully. In some cases, before entering underground, the investigator must conduct a survey of the employees of the emergency site and the persons exercising supervision and control over the observance of safety measures on it. 
At the third stage of preparation for the examination, the investigator sets the boundaries of the examination directly in the scene of the incident and outlines the sequence of its conduct and, depending on this, adjusts the obligations of the participants in the examination.

The working stage of the inspection should begin with the review of the scene of the incident [7]. At this stage, the investigator establishes the main landmarks, and the location of the incident is linked to the environment. At the same stage, it is advisable to conduct orientation and survey shooting and video recording of the scene in order to record its initial situation. If the boundaries of the inspection are not clear, then it is necessary to get specialist advice. After a general survey of the scene of the incident, the investigator proceeds to a direct examination of the objects associated with the scene.

In the static stage, the investigator examines the situation at the scene of the incident without any changes or movement of the objects being examined. At this stage, the investigator chooses the sequence of the examination - from the center to the periphery or from the periphery to the center [6]. It is impossible to give specific recommendations on the sequence of the inspection; the choice entirely depends on the situation at the scene of the incident. However, we believe that in this case, it is hardly advisable to conduct an inspection from the periphery to the center, since without being acquainted with the center of the scene, without getting an idea of the subject, scale, nature of the destruction, it is difficult to conduct a targeted search for evidence in the periphery. At this stage, the investigator must pay special attention to the state of technical means and devices. To this end, it is necessary to find out their name and purpose, marking data (type, model, serial number, date of manufacture), the position of the control manipulators and the condition of the sensors, and also fix all the defects and damages on them. Their position at the scene and their location relative to each other must be recorded.

In addition, the investigator must study the changes in the workings (collapses, large cracks), the state of the support, electrical facilities and carefully record in the protocol their condition, design, characteristics of the material from which they are made, correct installation and compliance with the requirements. Invited specialists play a significant role in displaying this information. When examining the surface in the emergency area, you should pay attention to the condition of the soil, its nature and slope, fix all traces on the ground.

Then the investigator proceeds to the dynamic stage of the inspection of the scene of the incident. Here the investigator examines in more detail both visible and hidden defects of mechanisms, machines, devices and assemblies. According to the tactical provisions of the inspection of the scene of the incident, the investigator at this stage can not only take the objects of inspection in his hands and move them in space, but also disassemble individual parts of the mechanisms and conduct a study of the density of adherence of various objects to each other.

The specificity of the inspection of the scene of the accident in the conditions of mining (determined by the production technology, the type of mining, the type of work performed and the use of machines and mechanisms, the nature and type of the accident) necessitates considering the tactics of inspecting the scene, depending on the type of violation of safety rules. However, given the limited scope of the article, we will focus on a brief description of the tactics of inspecting the scene when investigating methane explosions and fires.

This type of accidents is the most common and most dangerous both for people working in the mine and for the entire mine facility. Its danger is that combustion occurs in a confined space, and the products of combustion quickly spread through the workings. [9]

The specificity of the investigation of these crimes lies in the fact that after an accident, during the elimination of its consequences, the trace picture often changes significantly. The fire itself can arise and proceed not only in the workings where people work, but also in any other workings. 
Due to the specified specifics, when examining the scene of an incident associated with this type of accident, the investigator must study and record the ventilation method, the source of the formation of harmful gases and dust and the state of control over the mine air, the source of fire formation. When examining the state of ventilation in the emergency area, the investigator must take into account the gas content of the mine and the state of anti-dust measures.

When identifying the sources of the formation of explosive gases of coal dust, the investigator must take into account the fact that the release and accumulation of gases can proceed for a long time from the depth of the massif through various cracks and pores in the rock and coal seams, and the main places of accumulation of explosive gases are dead-end and ascending workings which should be carefully examined.

In Kuzbass mines, in addition to the accumulation of explosive gases, sudden emissions of coal and gas often occur. In this case, the investigator must study the procedure for coal seams development, the method of stripping, the methods mining and securing the preparatory and harvesting workings, as well as the implementation of real measures to prevent such emissions.

When examining the sources of gas and dust emission, the investigator should pay special attention to the following points: the state of mining of protective seams in relation to a hazardous seam; the methods used for drilling wells; advance by the bottom of the haulage drift of the working face; the method used to ventilate the mine; methods of drilling, excavation and loading of coal; properties and composition of coal dust; the use of special measures to combat coal dust (the presence and system of machines for irrigation and suction, cleaning, the use of rock dust distribution).

Of great importance for further investigation is the establishment of the zone of propagation of the explosion of gas and coal dust. During the inspection, special attention should be paid to determining the source of ignition of an explosive mixture, to traces of flame (soot) on the side surfaces of rocks, equipment, support, etc. The investigator should take into account that the source of ignition directly depends on the way the workings and coal are excavated. For example, in the case of using the hydraulic method, the use of explosives, electrical equipment and mechanical destruction of the coal seam are excluded, therefore, the first three sources cannot cause ignition.

During blasting operations in mines, shaking blasting takes place, which creates conditions for the release of coal and gas at the moment of the explosion, together with the presence of such components of the explosion as high temperature and open flame. Accordingly, the investigator must record not only what kind of explosive device was used, its design features; explosive, but also the type and method of explosion (instantaneous, short-delayed, delayed, multi-reception, etc.); the presence of chipped coal or rock cluttering the mine; presence of other power sources nearby; used drilling methods. It should be noted that the found remnants of explosive devices, in particular on the sleeve of the detonator cap, the electric detonator, on the shell of the cartridge, may retain fingerprints, which must be identified, fixed and seized.

Finding any electrical equipment or cables in the epicenter of the combustion is the basis for their detailed study during the inspection of the scene of the incident. The investigator must record the general position of the mine's power supply scheme - the location of electrical equipment, cables and the state of the power supply system. In our opinion, special attention should be paid to the study of the cable facilities. For this purpose, the investigator must clarify the routes and ways of laying cables, condition of cable glands, the state of production at the place where the cable is laid, the type and purpose of the cable, the state of the insulating sheath of the cable, the distance between the points of suspension and the methods used for hanging the cable, the methods of connecting cables with machines and devices and the state of these connections, the state of the joints of the pieces of cables, i.e. 
presence of metal liners or signs of hot vulcanization. Of no less importance is the identification of sections of current-carrying conductors of cable products of contact connections with reflow and other signs of emergency operation. All of the above circumstances are subject to mandatory recording in various ways.

At the same time, a plot of the electrical network must be drawn up for subsequent verification of the validity of the diagram provided by the mine management. If there are signs of any emergency processes on the cables, they are carefully recorded in the protocol of the investigative action, and then these sections of the cable are removed.

When inspecting electrical equipment, machines and mechanisms, it is necessary to pay attention to the condition of the insulation of machine parts, the condition of explosion-proof enclosures and the condition of their fastening, the presence and condition of grounding devices, the presence and condition of protective equipment, the position of buttons and control levers, the condition of local lighting and plug couplings, the position of the electric motor starters, the state and position of the emergency shutdown buttons for harvesters and cutting machines, the state of the applied interlocks. In addition, the compliance of the used electrical equipment with the level of explosion protection is being studied.

When inspecting magnetic starters used in mines, it is necessary to inspect not only the outer side of the starter, but also the inner one. When examining the outer side of the starters, the state of the point where the starter is installed, the state of the cable seal in the inputs, the presence of bolts, their number and their condition, damage on the cover itself, the presence of marking and other inscriptions are recorded. When examining the inside of the starter's lead-in box, attention is drawn to the state of grounding and its contact with the grounding screw, the presence of traces of melting of the cable cores, the presence of dust and moisture in it. When studying the starter mechanism, it is necessary to describe the tightness of the connection of the conductors to the terminals, the presence and condition of the electric extinguishing chamber, the condition of the working contacts, the presence and condition of the fuses, the rated current of the fuse-link, the presence of melting on live parts, the presence of dust, moisture, soot.

When inspecting all electrical facilities, the degree of their thermal damage is carefully studied and recorded. It should be noted that an inspection of the electrical equipment should be carried out in any case, even if the causes and the culprit of the accident are established.

The emergence of a fire source due to friction or collision occurs in accidents at mines less often than other sources. However, when examining the scene of the accident, the appearance of an open fire caused by friction cannot be ruled out.

During the inspection of the scene, if there is a suspicion of frictional occurrence of fire, the investigator should pay attention, first of all, to the type, size and mass of the machine or mechanism, data characterizing the load on those parts of the machine or mechanism that were subjected to friction, the speed of movement of the contacting bodies, the roughness of the contacting surfaces, the presence of lubricant, its type.

When inspecting machines and mechanisms, you should pay attention to the materials from which parts of certain objects are made, record the appearance of machines, mechanisms and their parts, which are supposedly exposed to frictional contact. And, if possible, seize such parts.

The next source of fire in the mine can be the appearance of open flame (hot work, smoking, etc.). For example, the prosecutor's office of the Novoilinsky district of the city of Novokuznetsk, Kemerovo region, opened a criminal case on the fact of smoking two workers of the Polosukhinskaya mine at their workplace.

Despite the fact that during the elimination of the accident, the situation at the scene of the incident significantly changes during the inspection, the investigator can detect objects, the presence of which indicates the use of open flames by the workers (cigarette butts, burnt matches, traces of bonfires, etc.). 
In practice, there are cases when unconventional situations are a source of open fire. For exzmple, during the investigation of the accident that occurred at the Zyryanovskaya mine in the city of Novokuznetsk, Kemerovo Region, it was found that the outbreak of methane gas and coal dust in longwall 14-01 at site No. 3 occurred as a result of mechanical destruction of the body (longitudinal rupture) of the mine insulating self-rescuer grade ShSS-1, which fell under the working 2GM-68B coal harvester. Mechanical impact on the body of the selfrescuer and the water and oil that got into it led to the combustion of the self-rescuer and an explosion of the gas-and-air mixture of methane and coal dust.

The emergence of endogenous fires on coal seams, prone to spontaneous combustion, leads to disruption of the technological process, the mine's dust and gas regime and possible accidents. One of the signs of such a fire is an increase in the content of carbon monoxide and hydrogen in the mine atmosphere. The occurrence of endogenous fires is associated with the stress-strain state of the rocks enclosing the mine workings. Therefore, work on coal seams prone to spontaneous combustion should be carried out strictly in accordance with the approved project.

When examining an emergency site with suspicion of an endogenous fire, the investigator should pay attention, first of all, to the presence of insulating bridges that prevent oxygen from entering the endogenous fire zone, and their condition, as well as signs of work to prevent spontaneous combustion (methods of fixing the overhanging coal mass with incombustible support, filling the voids behind the support with non-combustible materials, etc.).

Accidents caused by criminal violations of Safety Rules for mining operations are accompanied by human casualties. In accordance with paragraph 1 of Article 178 of the Criminal Procedure Code of the Russian Federation, the examination of the corpse at the place of its discovery is carried out by an investigator with the participation of a forensic expert. The tasks of a forensic expert when examining a corpse are: direct examination of the corpse; assisting the investigator in collecting material evidence of biological origin; giving recommendations to the investigator on the appointment of various types of forensic medical examinations.

On examination, two situations may arise: the corpse is at the place of its discovery and the corpse is raised to the surface. In any case, an investigator with a forensic medical expert acting as a specialist must conduct a thorough examination at the place of discovery of the corpse: its posture, the condition of the clothes and the contents of existing pockets, the presence of related items (self-rescuers, respirators, etc.), cadaveric changes, the presence of bodily injuries, their nature, localization, the mechanism of their formation (if possible). When examining the place where the corpse was found, it is necessary to study and record the location of the working, where the corpse was found and its exact location in this working; in addition, the objects on which the corpse lies are recorded.

Attention is drawn to the presence of traces of dragging a corpse, in cases where it may have been moved by someone working with the victim, and to the traces of a person's possible advancement before death, but after receiving bodily harm, which is associated with the person's ability to commit for any time active actions after receiving damage. This makes it possible to detect corpses at a considerable distance from the immediate scene of the incident. Mechanisms and machines located in the immediate vicinity of the discovered corpse must be recorded, with a special place given to the description of all protruding parts and details. As a result of the explosion, the body can be thrown away from the epicenter of the explosion, therefore, it is necessary to inspect the development along the entire possible movement path of the body from the explosion site to the place of its detection in order to detect clothing particles, traces of biological origin (blood, particles of skin and medulla, etc.), record the location of the corpse in relation to the epicenter of the explosion. In the case when the corpse is crushed, it is noted which part of the body is crushed, and by what object. 
The description of the posture of the corpse must begin in a strict sequence - from the general to the particular. First, the general position of the corpse on the surface, the posture of the corpse (some postures are characteristic of certain causes of death, for example, the "boxer" pose) are recorded, and then the position of the body, head, and limbs is described. In cases of death from mechanical destruction of the body as a result of an explosion, the position of each torn off piece of clothing and body in relation to the environment is described. Such thoroughness allows one to judge whether the corpse moved after death, and to put forward assumptions about the causes of death. It is imperative to record the presence of early cadaveric phenomena: cooling of the body, drying out of the mucous membranes, the location and color of cadaveric spots, and their color change depending on the dosed pressure on them and the time of color recovery, the stage of development of rigor mortis in various muscle groups.

In the case of examining the corpse of a person whose death occurred as a result of exposure to high temperatures (fire in a mine), special attention is paid to the localization of burns, burning of hair, signs of being alive in the fire (soot in the nasal passages, oral cavity, burns at the outer corners of the eyes - "screwing up effect"). When describing the corpse of a person whose death, presumably, occurred as a result of inhalation of combustion products, it is necessary to pay attention to the color of the cadaveric spots. Therefore, for example, pink-red, and not purple-violet, their color gives reason to talk about poisoning with carbon monoxide. When examining a corpse with suspicion of electrical injury, it is necessary to carefully examine the surface of the skin in order to identify electro markers and burns on it. This will provide an early indication of electric shock.

When describing clothes and shoes on a corpse, attention is drawn to their condition pollution, the presence of signs of thermal effects, smoking, the safety of clothes (absent or partially preserved in relation to the corresponding parts of the body), mechanical damage on it, possibly the presence of any characteristic odor. The contents of the pockets can give an idea of the possible cause of the accident (for example, smoking accessories). In the pockets of the victim's clothes there may be documents relevant to the case (task, technical documentation for the emergency area, etc.).

Putting related items (self-rescuers, respirators, etc.) on the record can allow the investigator to compose a picture of what happened. When fixing such items, the type of selfrescuer or respirator is described, attention is drawn to how the self-rescuer or respirator is put on, what is their condition (the presence of holes, seals, ruptures and contamination). The number of the self-rescuer or respirator is recorded, the presence of markings, seals, etc. on them. At the workplace, the victim can use rechargeable lighting devices. If they are available, the distance of such a lamp from the corpse is recorded, the inventory number is indicated and all existing damage is described, the position of the switches and how the lamp and the battery are attached to the victim's clothes are indicated.

When examining a corpse at the place of its discovery, the investigator must combine the recording of the results of such an examination in the protocol of the investigative action with the obligatory use of an investigative photo and video recording.

The large number of victims significantly increases the burden on forensic doctors. We believe that, in order to avoid mistakes by the investigator when drawing up a protocol for examining the scene of the incident when describing the corpses and the injuries they have on them, subsequently solving tasks to establish the causes and prescription of death by the forensic experts themselves, and other circumstances that determine the nature of the accident, a group of forensic doctors should be distinguished from 2-3 people (depending on the death toll).

The search and reconstructive activities of the investigator play the main role in achieving the general goals of inspecting the scene of the incident in such conditions. Therefore, the quality of search activity during an investigative action largely depends on psychological 
factors: its attitude to the examination of its participants, as well as their skills and abilities. Consequently, the effectiveness of the inspection will depend on the ability and skills of the investigator, his (her) desire to carry out painstaking work to search and find traces. Reconstructive activity will have a positive result only in the case of rational thinking of the investigator and a developed imagination.

\section{Conclusions}

1. High-quality inspection of the scene of the incident in the investigation of violations of safety rules in the conduct of mining operations depends on the clear organization of preparatory measures. One of the main actions of the investigator at the first stage of the preparatory work is to determine the circle of participants in the inspection of the scene. We believe that literal adherence to the prescription of the law on the participation of attesting witnesses in the inspection of the scene (except for the cases provided for in part 3 of article 170 of the Criminal Procedure Code of the Russian Federation) in the considered category of criminal cases is undesirable. The absence of attesting witnesses can be successfully compensated for by the use of technical means of recording, by expanding the circle of specialists - participants in the inspection of the scene of the incident.

2. It is advisable to expand the circle of specialists involved in the inspection of the accident site (electricians, mine surveyors, mine ventilation specialists, etc.).

3. A feature of the tactics of the working stage, in our opinion, is the use of an eccentric research method, regardless of the type of accident. At the same time, when directly searching for traces, carriers of information, moving from the center to the periphery, one should be guided, first of all, by the type of man-caused incident.

4. Given the complexity of recording the course and results of the investigative action directly at the inspection site, it is advisable, in our opinion, to draw up a report on the inspection of the scene of the incident in a draft version, and then prepare its final version on the surface, involving the appropriate specialists to accurately describe the objects and the situation on the place of the accident.

5. In order to avoid mistakes by the investigator when drawing up a protocol for examining the scene of the incident when describing the corpses and the injuries on them, subsequently solving tasks to establish the causes and prescription of death by the forensic experts themselves, and other circumstances that determine the nature of the accident, a group of forensic doctors should be distinguished from 23 people (depending on the number of victims) to assist the investigator in drawing up a protocol for examining the scene.

\section{References}

1. Crime Scene Investigation: A Guide for Law Enforcement (National Forensic Science Technology Center Largo, 2013)

2. S. Novoselov, V.Popov, Y. Filatov, H. Lee, A.Golik, E3S Web Conf. 21, 01034 (2017)

3. Y. Volgin, I. Gaag, A. Naumov, E3S Web Conf. 105, 02018 (2019)

4. The Constitution of the Russian Federation of December 12, 1993 (with subsequent amendments and additions), Collected Legislation of the Russian Federation, 9, 851 (2014)

5. The Criminal Procedure Code of the Russian Federation of December 18, 2001 No. 174FZ (with subsequent amendments and additions), Collected Legislation of the Russian Federation 1, 1 (2002)

6. O. Safargalieva, O. Sergeev, Y. Volgin, M. Agienko, E3S Web Conf. 105, 02018 (2019) 
7. M. Agienko, A. Naumov, I. Gaag, Y. Volgin, Web Conf. 41, 04046 (2018)

8. O. Safargalieva, O. Sergeev, Y. Volgin, M. Agienko, E3S Web Conf. 105, 02012 (2019)

9. V. F. Shchepelkov, N. I. Prjakhina, E.V. Suslina, Russian journal of criminology, 10(3), 598-607 (2016) 